
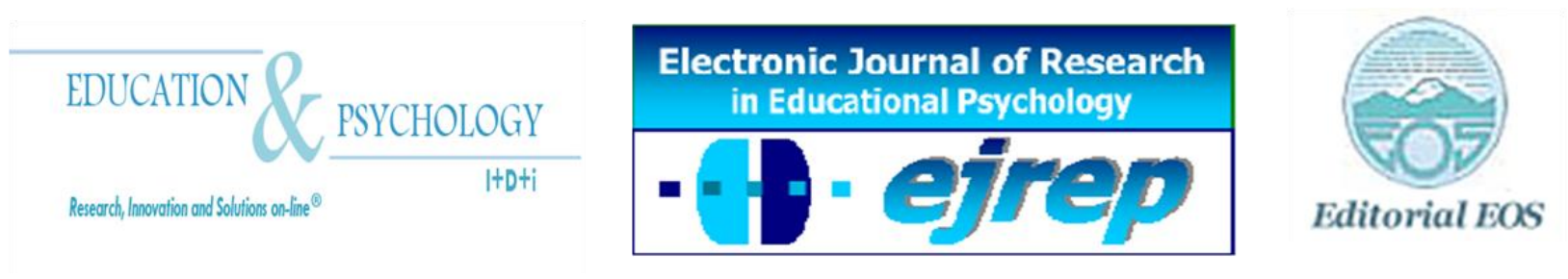

\title{
Meditación en Conciencia Plena: Una Nueva Aproximación para Reducir el Prejuicio
}

\author{
Lucía López-Rodríguez ${ }^{1}$, Marisol Navas ${ }^{2}$, \\ Clemente Franco Justo ${ }^{3}$, Israel Mañas Mañas ${ }^{4}$
}

${ }^{1}$ Departamento de Ciencias Humanas y Sociales, Universidad de Almería

${ }^{2}$ Departamento de Ciencias Humanas y Sociales, Universidad de Almería

${ }^{3}$ Departamento de Psicología Evolutiva y de la Educación, Universidad de Almería

${ }^{4}$ Departamento de Personalidad, Evaluación y Tratamiento Psicológicos, Universidad de Almería

\section{España}

Correspondencia: Lucía López Rodríguez. Dpto. de Ciencias Humanas y Sociales. Facultad de Psicología.

Universidad de Almería. Cañada de San Urbano s/n. Almería, 04120. España. E-mail: lucialopezrod@ gmail.com

(C) Education \& Psychology I+D+i and Editorial EOS (Spain) 


\section{Resumen}

Introducción. La convivencia multicultural es uno de los temas más importantes en los nuevos retos que plantea la educación. Como respuesta a las limitaciones de los enfoques de reducción del prejuicio, se ha comenzado a afrontar este problema desde las terapias cognitivas y comportamentales de nueva generación basadas en el mindfulness (conciencia plena) y la aceptación. Este estudio analiza la influencia de un programa psicoeducativo de entrenamiento en conciencia plena (mindfulness) sobre la actitud prejuiciosa (en tres componentes) y la discriminación percibida hacia las personas marroquíes en una muestra de 46 estudiantes de $4^{\circ}$ curso de ESO.

Método. Los estudiantes españoles que participaron en el estudio fueron asignados aleatoriamente a un grupo experimental $(\mathrm{N}=23)$, que recibió la intervención en meditación mindfulness, y a un grupo control $(\mathrm{N}=23)$. El estudio presenta un diseño cuasiexperimental con tres fases: pretest, postest y seguimiento. El estadístico de contraste $U$ de Mann-Whitney se utilizó para comprobar si existían diferencias significativas entre el grupo control y experimental, y la prueba de Wilcoxon para comprobar si existían diferencias significativas entre las diferentes fases del estudio en ambos grupos.

Resultados. Se mostró una disminución del componente afectivo y conativo del prejuicio en el grupo experimental (vs. grupo control). Los participantes del grupo experimental mostraron menos emociones negativas, menos distancia social preferida hacia marroquíes, más emociones positivas y más percepción de discriminación hacia ese grupo al finalizar el programa de meditación (vs. línea base), y estos efectos fueron relativamente duraderos.

Discusión y Conclusión: Los resultados de este estudio ponen de manifiesto que los enfoques psicológicos de nueva generación basados en el mindfulness pueden representar una herramienta útil y alternativa para reducir la actitud prejuiciosa (sobre todo de sus componentes afectivos) hacia minorías étnicas. Este enfoque parece más adaptado a los adolescentes y al nuevo prejuicio implícito.

Palabras Clave: Meditación, Conciencia plena, Actitud prejuiciosa, Discriminación percibida. 


\title{
Mindfulness Meditation: A New Approach to Decrease Prejudice
}

\begin{abstract}
Introduction. The multicultural coexistence is one of the most important issues in the new educational challenges. In the face of the limitations of the frameworks of prejudice reduction, this problem begins to be addressed from the Cognitive and Behavioural Therapies based on mindfulness and acceptation. This study analyses the influence of a psycho-educational program of training in mindfulness on prejudicial attitude and the discrimination perceived towards Moroccans in a sample of 46 high school students of 4th Grade.
\end{abstract}

Method. The Spanish students who participated in the study were randomly assigned to an experimental group $(\mathrm{N}=23)$, who was involved in a meditation program, and a control group $(\mathrm{N}=$ 23). The study presents a cuasiexperimental design with three phases: pretest, postest, and follow-up. The Mann-Whitney test was used to assess whether there were significant differences between the control and the experimental group, whereas the Wilcoxon test was used in order to assess whether there were significant differences among different phases of the study, in both groups.

Results. Results showed a decrease of two components of prejudice attitude towards Moroccans in the experimental group (affective and connative) compared to the control group. Experimental group showed less negative emotions and less preferred social distance towards Moroccans, as well as more positive emotions, and more perceived discrimination towards this ethnic group when they finished the meditation program, compared to the pre-test levels. These effects were relatively permanent.

Discussion and Conclusion. These results show that the psychological frameworks of new generation based on mindfulness can represent an useful and alternative tool in order to decrease prejudice (especially their affective components) towards ethnic minorities. Moreover, this approach seems to be more adapted to teenagers and to new implicit prejudice.

Keywords: Meditation, Mindfulness, Prejudice attitude, Perceived discrimination 


\section{Introducción}

La convivencia multicultural que la inmigración conlleva se manifiesta de forma importante en la educación. Los nuevos procesos de aculturación que se producen en autóctonos e inmigrantes crean nuevas realidades, y cada vez más los protagonistas de estos fenómenos son los jóvenes. España está siendo testigo de un incremento de alumnos extranjeros escolarizados, y de los nuevos retos que plantea la convivencia intercultural de adolescentes que poseen un bagaje cultural muy diferente. Ante esta realidad, el estudio de las actitudes intergrupales y el desarrollo de nuevas intervenciones para facilitar una convivencia más fluida se hace imprescindible para las ciencias sociales. El presente trabajo profundiza en nuevos métodos para la reducción del prejuicio en adolescentes.

La mayoría de los psicólogos sociales considera el prejuicio como una actitud negativa hacia un grupo social o sus miembros por el simple hecho de pertenecer a ese grupo (p.e., definición clásica de Allport, 1954). Sin embargo, el prejuicio puede ser resultado de múltiples determinantes y, a su vez, puede manifestarse a diferentes niveles (i.e., cognitivo, afectivo, conativo), por lo que es muy difícil de erradicar.

De acuerdo con el modelo de actitud de los tres componentes (Eagly y Chaiken, 1993; Harding, Kutner, Proshansky y Chein, 1969), la actitud prejuiciosa se caracterizaría por un componente cognitivo (i.e., las creencias negativas que las personas tienen sobre los miembros de un grupo social), un componente afectivo (i.e., emociones negativas que se experimentan en relación con otros grupos sociales) y un componente conativo-conductual (i.e., la disposición del individuo a comportarse de forma negativa con los miembros del exogrupo). Este último componente se denomina "discriminación", y en ocasiones, ante la dificultad de medirlo directamente como conducta observable, se considera que la intención de conducta manifestada por una persona hacia el mantenimiento o evitación de relaciones con miembros del exogrupo (la distancia social preferida) podría considerarse un indicador de este componente.

En los últimos años, se ha comenzado a estudiar el prejuicio implícito como una actitud automática que se produciría por medio de la activación espontánea de asociaciones mentales que no tienen porqué ser aceptadas conscientemente por el actor que las experimenta 
(Devine, 1989), y que derivan de las continuas representaciones culturales sobre los grupos que hemos aprendido a lo largo de nuestra vida y a las que somos expuestos diariamente. De esta forma, según el modelo de la disociación (Devine, 1989, 1995), en las sociedades contemporáneas se produce un conflicto entre las respuestas prejuiciosas automáticas hacia el exogrupo y las creencias no prejuiciosas, más controladas, predominantes en las sociedades igualitarias.

Al ser una actitud fuertemente aprendida, el prejuicio es muy difícil de erradicar, y aunque en ocasiones se puedan inhibir las asociaciones cognitivas previamente aprendidas, no se consigue eliminarlas del todo (Rehfeldt y Hayes, 2000). La investigación sobre las causas del prejuicio ha avanzado de forma paralela a sus intentos de reducirlo. Hasta ahora, estos intentos han ido encaminados, entre otros aspectos, a la búsqueda de metas supraordenadas para reducir la interdependencia negativa entre los grupos (Sherif, Harvey, White, Hood y Sherif, 1961), la desconfirmación de estereotipos o la percepción homogénea del exogrupo a través del contacto intergrupal (Pettigrew, 1997), o la creación de categorías supraordenadas que permitan a los individuos recategorizarse en un grupo más amplio que supere la división endo-exogrupo (Dovidio, Gaertner, Niemann y Snider, 2001). Aunque muchos de estos principios hayan funcionado parcialmente, aún no existen fórmulas infalibles para la reducción del prejuicio. Precisamente como respuesta a las limitaciones de estos enfoques, algunos autores han comenzado a afrontar este problema desde las terapias cognitivas y comportamentales (Cognitive and Behavioral Therapies, CBTs) de nueva generación basadas en el mindfulness (conciencia plena) y la aceptación.

Así, desde la teoría del marco relacional (RFT, Relational Frame Theory; Hayes, Barnes-Holmes y Roche, 2001) el prejuicio supone la objetivación de los seres humanos debido a su pertenencia a categorías verbales evaluativas, por las que se produce una transformación de funciones de las personas que pertenecen a un grupo social a causa del contacto directo o verbal con las funciones de unos pocos miembros de ese grupo social. Esta transformación de funciones se basa, más que en la experiencia directa, en las relaciones derivadas entre individuos particulares y la categoría grupal a la que pertenecen (Hayes et al., 2001).

De esta forma, las cogniciones que mantiene una persona con una psicopatología o con prejuicio son diferentes sólo aparentemente, pero en el fondo un pensamiento "prejuicioso" 
no es muy diferente a un pensamiento depresivo (Lillis y Hayes, 2007), ya que ambos se basan en funciones derivadas de otros estímulos. Esta idea justificaría la aplicación de estas nuevas terapias, basadas en el mindfulness y la aceptación, para la reducción del prejuicio exogrupal.

Algunos investigadores (p.e., Kucsera, 2009; Langer, Bashner y Chanowitz, 1985; Langer y Moldoveanu, 2000; Lillis y Hayes, 2007) han destacado los posibles beneficios del procesamiento mindfulness en la reducción de los estereotipos y el prejuicio hacia diversos grupos (p.e., étnicos, discapacitados, etc). En este sentido, Orr (2002) concluye que el mindfulness tiene la potencia de manejar el pensamiento dualista y fomentar el cambio cognitivo y afectivo.

Recientemente, Lillis y Hayes (2007) realizaron un estudio preliminar para comprobar si la terapia de aceptación y compromiso (ACT, Acceptance and Commitment Therapy) podría ser de utilidad para reducir el prejuicio étnico. A través de debates y ejercicios experienciales, los participantes deberían volverse más conscientes de sus propios pensamientos, sentimientos y reacciones prejuiciosas, aceptarlos como resultado natural de su socialización en una sociedad prejuiciosa, y percatarse de los procesos automáticos de evaluación que ponían en marcha, para finalmente orientarse hacia acciones positivas coherentes con sus propios valores. Sus resultados mostraron que en la condición de entrenamiento con ACT, las intenciones de acción positiva hacia otros grupos culturales y la aceptación y flexibilidad de pensamientos prejuiciosos mejoraron significativamente (desde el pretest al postest) en comparación con la condición de formación educativa sobre toma de conciencia del prejuicio, y que estos cambios se mantenían durante un periodo de seguimiento a corto plazo. También demostraron que la aceptación y la flexibilidad parcialmente mediaban el impacto de la ACT sobre las intenciones de acción positiva. Según los autores, los estudiantes se volvieron más capaces de reconocer sus propios procesos de evaluación social, prestaron menos atención al contenido de sus pensamientos, y así sus comportamientos se hicieron más flexibles. Para los autores, estos nuevos enfoques podrían suponer una alternativa o un complemento a las intervenciones basadas en el contacto, para conseguir efectos más consistentes (Lillis y Hayes, 2007).

A pesar de la novedad de aplicar estas nuevas terapias a la reducción del prejuicio, la idea de tomar conciencia de nuestras actitudes prejuiciosas para disminuir el prejuicio ya fue 
abordada por Devine (1989), quien mantiene que tanto las personas prejuiciosas como las no prejuiciosas activan automáticamente los estereotipos culturales sobre los grupos minoritarios. Sin embargo, lo que diferencia a unas y otras es que las poco prejuiciosas se encuentran más motivadas para contrarrestar sus reacciones prejuiciosas espontáneas. Algunos estudios (p.e., Devine y Monteith, 1993) muestran cómo la conciencia sobre la inconsistencia entre el comportamiento interétnico y los estándares igualitarios conscientes, produce una reacción emocional negativa en las personas que puede desencadenar por sí sola una mayor motivación para controlar las respuestas prejuiciosas espontáneas, y de esta forma, comportarse de una forma más igualitaria con el exogrupo.

Esta idea de tomar conciencia y aceptar lo que realmente pensamos y sentimos como una forma de reducir la actitud negativa hacia grupos minoritarios conecta perfectamente con la ACT y la meditación a través del desarrollo del mindfulness. Aunque con una diferencia clara: en estas intervenciones no se intenta controlar lo que pensamos y sentimos, ya que según esta perspectiva esto produciría el efecto paradójico de anclarnos más en esos pensamientos y sentimientos.

El presente estudio utiliza precisamente un programa psicoeducativo de entrenamiento en conciencia plena (mindfulness) con el objetivo de comprobar sus efectos en los tres componentes de la actitud prejuiciosa y la percepción de discriminación hacia las personas marroquíes por parte de estudiantes de Educación Secundaria Obligatoria (ESO). Se eligió el exogrupo marroquí por su alta representatividad en la provincia donde se desarrolla la investigación (Almería, España), ya que constituye el grupo extranjero más numeroso (el 5.98\% de la población según el Avance del Padrón a 1 de enero de 2010; INE, 2010). El estudio utiliza un diseño cuasiexperimental, con un grupo experimental y un grupo control con medición pretest-postest-seguimiento.

\section{Método}

\section{Participantes}

Participaron en la investigación 46 estudiantes de cuarto curso de ESO pertenecientes a un centro público de la provincia de Almería (España). Sus edades oscilaban entre los 15 y 
los 18 años $(M=16.8 ; D T=1.04)$. El grupo experimental estuvo formado por 23 alumnos (14 chicas y 9 chicos), y el grupo control por otros 23 alumnos (16 chicas y 7 chicos).

\section{Instrumentos}

Actitud prejuiciosa. Se midió con el Test de Actitud Prejuiciosa (TAP; Navas y Rojas, 2010; Rojas, Lozano, Navas y Pérez, 2011), adaptado para medir prejuicio hacia personas marroquíes, y compuesto por tres subescalas que constituyen indicadores de los tres componentes de la actitud prejuiciosa: cognitivo, afectivo y conativo-conductual.

En el componente cognitivo los participantes tenían que valorar una serie de ámbitos del exogrupo (político, bienestar social, laboral, económico, social, familiar, religioso y de valores), utilizando una escala de respuesta que oscilaba entre 1 (muy malo) y 5 (muy bueno), cuyas puntuaciones fueron invertidas, de manera que una alta puntuación indicaba una peor opinión del exogrupo (i.e., mayor prejuicio), y finalmente promediadas.

El componente afectivo de la actitud prejuiciosa se midió a través de tres tipos de emociones: positivas (admiración, simpatía y respeto), negativas sutiles (desconfianza, incomodidad, inseguridad e indiferencia), y negativas antiguas (irritación, odio, desprecio y miedo), con el objetivo de analizar sus posibles diferencias o especificidades. Los participantes debían indicar "el grado en el que habían sentido cada una de esas emociones" hacia las personas marroquíes, utilizando una escala de respuesta que oscilaba entre 1 (nada) y 5 (mисho). Las puntuaciones en las emociones que conformaban cada dimensión fueron promediadas.

Finalmente el componente conativo-conductual de la actitud prejuiciosa se midió con una escala clásica de distancia social (p.e., Bogardus, 1927), que medía el tipo de relación (cercana o distante) que los participantes preferían mantener con personas marroquíes. Se midió con un sólo ítem ("Si pudiera elegir, ¿hasta dónde estaría Vd. dispuestola a llegar en su relación con personas marroquíes?") con 5 alternativas de respuesta, que oscilaban desde la preferencia por mantener una relación muy íntima con estas personas hasta la preferencia por la ausencia de relación con ellas, pasando por opciones intermedias. Los participantes podían señalar varias opciones, por lo que se utilizó para los análisis la opción más inclusiva (la que indicaba una preferencia por mantener una relación más íntima con el exogrupo), 
indicando las puntuaciones altas una preferencia por mantener más distancia social con el exogrupo (i.e., mayor prejuicio).

El TAP mostró una fiabilidad aceptable, ya que el coeficiente alpha de Cronbach fue de .76 para el componente cognitivo, de .89 para emociones positivas, .81 para emociones negativas sutiles, .95 para emociones negativas antiguas (.93 para el componente afectivo), y de .88 para el componente conativo-conductual.

Discriminación percibida. Hace referencia a la percepción de los autóctonos sobre el grado de discriminación que sufren los inmigrantes en nuestro país ("grado en el que son peor tratados que los españoles en diferentes ámbitos"). Para medirla se utilizaron 7 ítems (Navas y Rojas, 2010), cada uno de ellos correspondiente a un ámbito posible de discriminación (i.e., político, sistema de bienestar social, vivienda, trabajo, social, medios de comunicación y religioso) que fueron promediados. Las alternativas de respuesta en cada ítem oscilaban entre 1 (nada) y 5 (mucho), por lo que a mayor puntuación en esta escala más percepción de que los miembros del exogrupo están siendo discriminados. El coeficiente de fiabilidad alpha de Cronbach fue de .84 .

\section{Procedimiento}

La muestra se obtuvo a través del contacto con los tutores de dos grupos de cuarto curso de ESO. A uno de ellos se le ofreció la posibilidad de impartir un taller de entrenamiento en conciencia plena dirigido a sus estudiantes en las horas de tutoría durante el segundo trimestre del curso. Al otro tutor se le pidió colaboración para aplicar a sus estudiantes los dos instrumentos ya descritos, en las diferentes fases del estudio.

Antes del comienzo del curso en el grupo experimental se obtuvo la medida pretest de la actitud prejuiciosa y la discriminación percibida hacia la población marroquí, para lo cual todos los participantes respondieron de forma individual al Test de Actitud Prejuiciosa (TAP) y a la Escala de Discriminación percibida.

Posteriormente, se aplicó el programa de intervención en el grupo experimental, realizándose una sesión semanal de una hora de duración a lo largo de 11 semanas, consistente en un programa psicoeducativo de meditación mindfulness para el desarrollo de la conciencia plena denominado Meditación Fluir (Franco, 2009). El aprendizaje de dicha 
técnica de meditación fue completado con la presentación y discusión de diversas metáforas y ejercicios utilizados en la terapia de aceptación y compromiso (Hayes, Stroshal y Wilson, 1999; Wilson y Luciano, 2002), junto con relatos de la tradición zen (Deshimaru, 2006) y de la meditación vipassana (Hart, 1994), los cuales tienen como objetivo, igual que el programa de meditación mindfulness, aprender a tomar distancia de los pensamientos, sentimientos y emociones, aprendiendo a observarlos de una manera imparcial, sin dejarnos involucrar o influir por su contenido.

Para facilitar la implementación del programa, los participantes tenían que realizar diariamente en casa dos de los ejercicios aprendidos previamente en el programa (recorrido corporal durante 10 minutos y práctica de conciencia plena sobre la respiración en la zona del abdomen durante 15 minutos), así como completar a lo largo de la semana una hoja de autoregistro en la que diariamente debían anotar si habían practicado los ejercicios. El grado medio de cumplimiento de estos ejercicios fue del 59\% (recorrido corporal) y 64\% (conciencia plena). No se tuvieron en cuenta en los análisis a aquellos participantes que acudieron a menos del 50\% de las sesiones del programa de intervención, y que en la hoja de auto-registro informaron haber practicado menos del 50\% de los días ambos ejercicios.

Una vez finalizado el programa de intervención se obtuvieron las medidas postest, administrando nuevamente a los participantes las dos escalas descritas. Tres meses después de haber finalizado la intervención se realizó una medida de seguimiento con las mismas escalas. Todos los participantes en el estudio fueron informados al finalizar éste del objetivo de la investigación, y se solicitó su consentimiento por escrito, manteniendo y garantizando la confidencialidad y el anonimato.

\section{Análisis de Datos}

Para comprobar la existencia de diferencias estadísticamente significativas entre las puntuaciones medias del grupo control y experimental en cada una de las fases del estudio para la actitud prejuiciosa y la discriminación percibida hacia la población marroquí, se empleó el estadístico de contraste no paramétrico $U$ de Mann-Whitney para muestras independientes, ya que los datos no se ajustaban a la distribución normal.

Para comprobar la existencia de diferencias estadísticamente significativas en ambas variables, en cada una de las fases del estudio, en ambos grupos se empleó el estadístico de 
contraste no paramétrico de Wilcoxon para muestras relacionadas, ya que los datos no se ajustaban a la distribución normal.

Finalmente, para valorar la magnitud del cambio experimentado tras la intervención, tanto en la medida postest como en la medida de seguimiento en el grupo experimental, se empleó la $d$ de Cohen (Cohen, 1988) - valores superiores a 0.8 indican cambios muy importantes, entre 0.5 y 0.8 importantes, por debajo de 0.5 medios y menores de 0.2 bajos -, así como el porcentaje de cambio entre las puntuaciones pretest-postest y pretest-seguimiento.

\section{Resultados}

Las medias y desviaciones típicas para los componentes de la actitud prejuiciosa y la discriminación percibida correspondientes a los grupos experimental y control en cada una de las fases del estudio se encuentran en la Tabla 1.

Tabla 1. Medias y Desviaciones Típicas Pretest, Postest y Seguimiento en los componentes de la actitud prejuiciosa y la discriminación percibida. Grupo Experimental y Grupo Control

\begin{tabular}{lcccccc}
\hline \multicolumn{1}{c}{ Grupo Experimental } & & & \\
\hline \multicolumn{1}{c}{ Pariable } & $M$ & $D T$ & $M$ & $D T$ & $M$ & $D T$ \\
\hline Comp. Cognitivo & 2.37 & $(0.81)$ & 2.48 & $(0.66)$ & 2.45 & $(0.74)$ \\
Emoc. Positivas & 2.89 & $(0.78)$ & 3.39 & $(0.96)$ & 3.21 & $(0.93)$ \\
Emoc. Negat. Sutiles & 2.77 & $(0.95)$ & 2.09 & $(0.83)$ & 2.17 & $(0.84)$ \\
Emoc. Negat. Antiguas & 2.23 & $(0.89)$ & 1.84 & $(0.95)$ & 2.03 & $(0.72)$ \\
Distancia Social & 2.69 & $(0.98)$ & 2.23 & $(1.01)$ & 2.38 & $(1.04)$ \\
Discriminación Percibida & 2.86 & $(0.91)$ & 3.27 & $(0.93)$ & 3.13 & $(0.84)$ \\
\hline
\end{tabular}




\begin{tabular}{lccccccc}
\hline & \multicolumn{2}{l}{ Grupo Control } & & & \\
\hline \multicolumn{1}{c}{ Pariable } & $M$ & $D T$ & $M$ & $D T$ & $M$ & $D T$ \\
\hline \multicolumn{1}{c}{ Prest } & \multicolumn{2}{c}{ Postest } & \multicolumn{2}{c}{ Seguimiento } \\
& 2.23 & $(0.87)$ & 2.33 & $(0.79)$ & 2.17 & $(1.01)$ \\
\hline Comp. Cognitivo & 2.95 & $(1.01)$ & 3.02 & $(0.93)$ & 3.06 & $(0.92)$ \\
Emoc. Positivas & 3.05 & $(1.13)$ & 3.15 & $(1.04)$ & 3.17 & $(0.84)$ \\
Emoc. Negat. Sutiles & 2.27 & $(0.92)$ & 2.88 & $(0.83)$ & 2.98 & $(0.82)$ \\
Emoc. Negat. Antiguas & 2.92 & $(1.44)$ & 3.00 & $(1.35)$ & 3.08 & $(1.25)$ \\
Distancia Social & 2.83 & $(0.99)$ & 2.82 & $(1.03)$ & 2.74 & $(0.82)$ \\
Discriminación Percibida & & & & & & \\
\hline
\end{tabular}

Las pruebas no paramétricas $U$ de Mann-Whitney para muestras independientes indicaron que no existían diferencias significativas de partida entre las puntaciones del grupo experimental y control en las variables del estudio (ver Tabla 2). Sin embargo, sí aparecieron diferencias entre ambos grupos en el postest: el grupo experimental mostró significativamente menos emociones negativas sutiles y antiguas y más emociones positivas (componente afectivo del prejuicio), menos distancia social (componente conativo) y más discriminación percibida, en comparación con el grupo control. Las mismas diferencias entre el grupo experimental y control se mantienen en la fase de seguimiento excepto en emociones positivas.

Tabla 2. Prueba U de Mann-Whitney para Muestras Independientes de las Diferencias Pretest, Postest y Seguimiento entre Grupo Control y Experimental en los componentes de actitud prejuiciosa y discriminación percibida.

\begin{tabular}{lcccccc}
\hline & \multicolumn{2}{c}{ Pretest } & \multicolumn{2}{c}{ Postest } & \multicolumn{2}{c}{ Seguimiento } \\
\hline \multicolumn{1}{c}{ Variable } & $z$ & $p$ & $z$ & $p$ & $z$ & $p$ \\
\hline Comp. Cognitivo & 1.15 & .264 & 1.27 & .148 & 1.07 & .185 \\
Emoc. Positivas & .67 & .616 & 2.43 & $.039 *$ & .823 & .235 \\
Emoc. Negat. Sutiles & .69 & .495 & 3.00 & $.006 * *$ & 3.24 & $.003 * * *$ \\
Emoc. Negat. Antiguas & .23 & .813 & 3.31 & $.003 * * *$ & 3.37 & $.003 * * *$ \\
Distancia Social & .42 & .674 & 2.96 & $.008^{* *}$ & 2.99 & $.008^{* *}$ \\
Discriminación Percibida & .54 & .591 & 2.74 & $.016^{*}$ & 2.65 & $.021^{*}$ \\
\hline Nota: *** $p<.005 * * p<.01 * * p<05$ & & & & & &
\end{tabular}


Las pruebas no paramétricas de Wilcoxon para muestras relacionadas indicaron que en el grupo experimental existían diferencias estadísticamente significativas al comparar las puntuaciones pretest-postest: después de la intervención los participantes mostraron significativamente menos emociones negativas sutiles y antiguas, y más emociones positivas (componente afectivo), menos distancia social (componente conativo), y significativamente más discriminación percibida (vs. puntuaciones pretest). En este grupo también se produjeron diferencias entre el pretest-seguimiento, observándose significativamente menos emociones negativas sutiles y distancia social, y un incremento significativo en emociones positivas y discriminación percibida en la fase de seguimiento en comparación con la fase pretest (Ver Tabla 3).

En el caso del grupo control, no se observan diferencias estadísticamente significativas al realizar las comparaciones pretest-postest y pretest-seguimiento, con la excepción de las emociones negativas antiguas, que se incrementan significativamente en el postest y en el seguimiento en comparación con la fase pretest (ver Tabla 3).

Tabla 3. Prueba de Wilcoxon para Muestras Relacionadas de las Diferencias Pretest-Postest, Pretest-Seguimiento y Postest-Seguimiento en los componentes de actitud prejuiciosa y discriminación percibida. Grupo Experimental y Grupo Control

\begin{tabular}{|c|c|c|c|c|c|c|}
\hline \multicolumn{7}{|c|}{ Grupo Experimental } \\
\hline & \multicolumn{2}{|c|}{ Pre-Post } & \multicolumn{2}{|c|}{ Pre-Seg. } & \multicolumn{2}{|c|}{ Post-Seg. } \\
\hline Variable & $z$ & $p$ & $z$ & $p$ & $z$ & $p$ \\
\hline Comp. Cognitivo & 1.84 & .065 & .97 & .329 & .69 & .490 \\
\hline Emoc. Positivas & 2.66 & $.021 *$ & 2.05 & $.047 *$ & 1.03 & .172 \\
\hline Emoc. Negat. Sutiles & -3.24 & $.001 * * *$ & -2.96 & $.003 * *$ & .89 & .372 \\
\hline Emoc. Negat. Antiguas & -3.02 & $.002 * *$ & -1.55 & .121 & 1.87 & .061 \\
\hline Distancia Social & -2.75 & $.014 *$ & -2.00 & $.046^{*}$ & 1.44 & .157 \\
\hline Discriminación Percibida & 2.78 & $.013 *$ & 2.64 & $.019 *$ & .81 & .414 \\
\hline
\end{tabular}




\begin{tabular}{|c|c|c|c|c|c|c|}
\hline \multicolumn{7}{|c|}{ Grupo Control } \\
\hline & \multicolumn{2}{|c|}{ Pre-Post } & \multicolumn{2}{|c|}{ Pre-Seg. } & \multicolumn{2}{|c|}{ Post-Seg. } \\
\hline Variable & $z$ & $p$ & $z$ & $p$ & $z$ & $p$ \\
\hline Comp. Cognitivo & .91 & .927 & .06 & .952 & .48 & .627 \\
\hline Emoc. Positivas & .28 & .776 & 1.05 & .290 & .37 & .710 \\
\hline Emoc. Negat. Sutiles & 1.05 & .290 & .96 & .337 & .25 & .803 \\
\hline Emoc. Negat. Antiguas & 2.36 & $.018 *$ & 2.62 & $.009 * *$ & 1.11 & .265 \\
\hline Distancia Social & .57 & .564 & .70 & .480 & .57 & .564 \\
\hline Discriminación Percibida & .072 & .942 & .28 & .779 & .46 & .641 \\
\hline
\end{tabular}

Las puntuaciones de la $d$ de Cohen para las medidas postest mostraron la existencia de cambios importantes en las emociones negativas sutiles $(d=.76)$ y en las emociones positivas $(d=.57)$; cambios de nivel medio en las emociones negativas antiguas $(d=.42)$, distancia social $(d=.46)$ y en discriminación percibida $(d=.45)$; y cambios bajos en el componente cognitivo del prejuicio $(d=.15)$ (ver Tabla 4$)$.

Por lo que respecta a la magnitud del cambio experimentado entre las puntuaciones pretest-seguimiento, se observa la existencia de cambios importantes en emociones negativas sutiles $(d=.67)$; cambios medios en emociones positivas $(d=.37)$, en distancia social $(d=$ $.31)$, en emociones negativas antiguas $(d=.25)$ y en discriminación percibida $(d=.31)$; y cambios bajos en el componente cognitivo $(d=.10)$. 
Tabla 4. d de Cohen y Porcentaje de Cambio Pretest-Postest y Pretest-Seguimiento en los componentes de la actitud prejuiciosa y la discriminación percibida en el Grupo Experimental

\begin{tabular}{lcccc}
\hline \multicolumn{1}{c}{ Variable } & \multicolumn{2}{c}{ Pretest-Postest } & \multicolumn{2}{c}{$\begin{array}{c}\text { Pretest- } \\
\text { Seguimiento }\end{array}$} \\
\hline Comp. Cognitivo & .15 & 4.64 & .10 & 3.37 \\
Emoc. Positivas & .57 & 17.31 & .37 & 11.07 \\
Emoc. Negat. Sutiles & .76 & -24.5 & .67 & -21.6 \\
Emoc. Negat. Antiguas & .42 & -17.5 & .25 & -9.9 \\
Distancia Social & .46 & -17.1 & .31 & -11.5 \\
Discriminación Percibida & .45 & 14.33 & .31 & 9.44 \\
\hline
\end{tabular}

Respecto al porcentaje de cambio pretest-postest, se muestra una reducción del $24.5 \%$ para emociones negativas sutiles, del $17.5 \%$ para emociones negativas antiguas, del $17.1 \%$ para la distancia social, y un incremento del $17.31 \%$ para las emociones positivas. Se muestra también un incremento del $14.33 \%$ para la discriminación percibida, y un limitado $4.64 \%$ para el componente cognitivo del prejuicio.

Por otra parte, el porcentaje de cambio experimentado entre las puntuaciones pretestseguimiento muestra una reducción del $21.7 \%$ para emociones negativas sutiles, del $9.9 \%$ para emociones negativas antiguas, del $11.5 \%$ para la distancia social, e incremento del $11 \%$ para emociones positivas. Se muestra también un incremento del 9.4\% para la discriminación percibida, y un limitado $3.37 \%$ para el componente cognitivo del prejuicio.

\section{Discusión y conclusiones}

Los resultados de este estudio ponen de manifiesto que los enfoques psicológicos de nueva generación basados en el mindfulness pueden representar una herramienta útil y alternativa para reducir la actitud prejuiciosa (o al menos algunos de sus componentes) hacia minorías étnicas. Así, aquellos participantes que trabajaron con el programa psicoeducativo de meditación mindfulness mostraron hacia las personas de origen marroquí significativamente menos emociones negativas sutiles y antiguas, más emociones positivas y discriminación percibida, y menos distancia social que el grupo que no realizó esta formación. 
Asimismo, a nivel intra-participante puede observarse cómo los jóvenes que realizaron el programa de meditación mindfulness experimentaron cambios en sus propios niveles de prejuicio tras la intervención, mostrando significativamente menos emociones negativas sutiles y antiguas, más emociones positivas, menos distancia social preferida hacia marroquíes, así como más discriminación percibida hacia este grupo.

Estos efectos son consistentes con los resultados encontrados por Lillis y Hayes (2007) en su estudio previo sobre el efecto de la ACT en la reducción de actitudes prejuiciosas. Asimismo, permiten constatar que el entrenamiento en mindfulness puede tener un efecto incluso sobre las intenciones de conducta, ya que los participantes reconocieron que estarían dispuestos a mantener relaciones más estrechas con los inmigrantes marroquíes (i.e., menos distancia social).

En cuanto a la interpretación de estos efectos, los resultados de nuestro estudio pueden estar poniendo de manifiesto un proceso de descategorización social, como reconocieron Lillis y Hayes (2007) analizando los resultados de sus estudios. Los participantes podrían haber reducido las diferencias entre las categorías sociales ellos-nosotros, focalizándose con conciencia plena y sin juicios de valor en sus procesos de sentimiento prejuicioso, en lugar de en el contenido de esos sentimientos, reduciendo por lo tanto su impacto sobre la conducta (Lillis y Hayes, 2007), por lo que se tendería a disminuir también la distancia social preferida entre ellos y nosotros.

Resulta destacable que los cambios más importantes se produzcan en el componente afectivo de la actitud prejuiciosa, mientras que los cambios en el componente cognitivo del prejuicio apenas ocurren. Estos resultados son consistentes con otros enfoques de reducción del prejuicio, como la teoría del contacto (Pettigrew, 2008). En ella se contemplan mediadores cognitivos y afectivos de los efectos del contacto, pero parece que los segundos son mucho más importantes que los primeros, por lo que el efecto del contacto intergrupal reduciría los componentes afectivos del prejuicio más que sus componentes cognitivos (Pettigrew, 1997; Tropp y Pettigrew, 2005). En sintonía con esta teoría, en el presente estudio la meditación mindfulness parece actuar más sobre el componente afectivo (en tres tipos de emociones: positivas, negativas sutiles y negativas antiguas) o conativo del prejuicio que sobre su componente cognitivo. 
Una idea interesante que puede derivarse de los resultados es que este efecto es duradero a corto plazo (seguimiento: tres meses) en relación a las emociones negativas sutiles y la distancia social (que se mantienen más bajas que en el pretest) y a las emociones positivas y la discriminación percibida (más altas que en el pretest) en el grupo experimental. Un aspecto importante del estudio es que se lleva a cabo en un contexto real lo que facilitaría a los participantes la generalización de las nuevas funciones aprendidas. No obstante, el estudio adolece de algunas limitaciones, tales como la imposibilidad de identificar qué causó realmente esta mejora actitudinal, ya que la intervención estaba compuesta por varios elementos diferentes. Aunque el resultado es positivo, ya que todos en su conjunto produjeron efectos de reducción de las actitudes prejuiciosas hacia los marroquíes, sería adecuado realizar estudios que permitieran desvelar qué aspecto de la intervención potencia más este efecto. Asimismo, otra limitación se relaciona con el grupo control, que no realizó ningún programa psicoeducativo, por lo que, aunque el estudio nos indica que esta intervención es útil, deberían realizarse comparaciones con otro tipo de intervenciones, para explorar su eficacia comparada con otros métodos de reducción del prejuicio.

Sin embargo, a pesar de sus limitaciones, este estudio confirma la idea de que otras técnicas alternativas como la meditación basada en el mindfulness y las técnicas de la ACT pueden ser muy útiles en la reducción de las actitudes prejuiciosas hacia grupos étnicos minoritarios, especialmente en su componente afectivo, que como muestra la literatura, parece ser uno de los aspectos más importantes en el prejuicio. Además, este trabajo contribuye al desarrollo de nuevas aplicaciones de la ACT ampliando la intervención a ámbitos más educativos y psicosociales.

La reducción del prejuicio en ámbitos educativos es esencial para la creación de un futuro de tolerancia y multiculturalidad. En este sentido, los hallazgos del presente trabajo enfatizan la importancia de buscar nuevas formas para fomentar relaciones interculturales armoniosas más adaptadas a los adolescentes y al nuevo prejuicio implícito. 


\section{Agradecimientos}

Esta investigación ha sido parcialmente desarrollada dentro del proyecto de investigación financiado por la Consejería de Empleo (Dirección General de Coordinación de Políticas Migratorias; Junta de Andalucía; Referencia 2009/217), y del Programa FPU del Ministerio de Educación y Ciencia, España (Referencia AP2009-4881).

\section{Referencias}

Allport, G. W. (1954). The Nature of Prejudice. Cambridge, Mass: Addison-Wesley.

Bogardus, E. S. (1927). Race friendliness and social distance. Journal of Applied Sociology, 11, 272-287.

Cohen, J. (1988). Statistical Power Analysis for the Behavioral Sciences (2a ed.). Nueva Jersey: Lawrence Erlbaum Associates.

Deshimaru, T. (2006). La práctica del zen. Barcelona: RBA.

Devine, P. G. (1989). Stereotypes and prejudice: Their automatic and controlled components. Journal of Personality and Social Psychology, 56, 5-18.

Devine, P. G. (1995). Prejudice and the perception of outgroups. En A. Tesser (Ed.), Advanced Social Psychology (pp. 467-524). New York: McGraw-Hill.

Devine, P. G. y Monteith, M. J. (1993). The role of discrepancy-associated affect in prejudice reduction. En D. M. Mackie y D. L. Hamilton (Eds.), Affect, cognition, and stereotyping: Interactive processes in intergroup perception (pp. 317-344). Orlando, FL: Academic Press.

Dovidio, J. F., Gaertner, S. L., Niemann, Y. F. y Snider, K. (2001). Racial, ethnic, and cultural Differences in responding to distinctiveness and discrimination on campus: Stigma and common group identity. Journal of Social Issues, 57, 167-188.

Eagly, A. y Chaiken, S. (1993). Psychology of Attitudes. NY: Harcourt, Brace Jovanovich.

Franco, C. (2009). Meditación Fluir para serenar el cuerpo y la mente. Madrid: Bubok.

Harding, J., Kutner, B. Proshansky, H. y Chein, I. (1969). Prejudice and ethnic relations. En G. Lindzey y E. Aronson (Eds.), Handbook of Social Psychology (pp.1-76). Reading: Addison Wesley.

Hart, W. (1994). La Vippasana. El arte de la meditación. Madrid: Luz de Oriente. 
Hayes, S. C., Barnes-Holmes, D. y Roche, B. (2001). Relational frame theory: A postskinnerian account of human language and cognition. Nueva York: Kluwer/Plenum.

Hayes, S. C., Stroshal, K.D. y Wilson, K.G. (1999). Acceptance and Commitment Therapy. New York: The Guilford Press.

Instituto Nacional de Estadística (2010). Avance del Padrón a 1 de enero de 2010. Recuperado del Instituto Nacional de Estadística en:

http://www.ine.es/jaxi/menu.do?type=pcaxis \&file=pcaxis\&path=\%2Ft20\%2Fe245\%2Fp04\%2F\%2Fa2010

Kucsera, J.V. (2009). Racial Mindfulness: Exploring the Influence of Mindfulness on Racial Biases. Tesis publicada en University of Texas (Austin).

Langer, E., Bashner, R. y Chanowitz, B. (1985). Decreasing prejudice by increasing discrimination. Journal of Personality and Social Psychology, 49, 113-120.

Langer, E. y Moldoveanu, M. (2000). The construct of mindfulness. Journal of Social Issues, $56,1-9$.

Lillis, J. y Hayes, S. C. (2007). Applying acceptance, mindfulness, and values to the reduction of prejudice: A pilot study. Behavior Modification, 31, 389-411.

Navas, M. S. y Rojas, A. J. (Coords.) (2010). Aplicación del Modelo Ampliado de Aculturación relativa (MAAR) a nuevos colectivos de inmigrantes en Andalucía: rumanos y ecuatorianos. Sevilla: Consejería de Empleo (Junta de Andalucía).

Orr, D. (2002). The uses of mindfulness in ant-oppressive pedagogies: Philosophy and praxis. Canadian Journal of Education, 27, 477-490.

Pettigrew, T. F. (1997). Generalized intergroup contact effects on prejudice. Personality and Social Psychology Bulletin, 23, 173-185.

Pettigrew, T.F. (2008). Future directions for intergroup contact theory and research. International Journal of Intercultural Relations, 32, 187-199.

Rehfeldt, R. A. y Hayes, L. J. (2000). The long-term retention of generalized equivalence glasses. Psychological Record, 50, 405-428.

Rojas, A.J., Lozano, O., Navas, M.S. y Pérez, P.J. (2011). Prejudiced Attitude Measurement using the Rasch Rating Scale Model. Psychological Reports, 109 (2), 553-572

Sherif, M., Harvey, O. J., White, B. J., Hood, W. R. y Sherif, C. W. (1961). Intergroup cooperation and competition: The Robbers Cave Experiment. Norman: Institute of Group Relations, University of Oklahoma. 
Tropp, L. R. y Pettigrew, T. F. (2005). Differential relationships between intergroup contact and affective and cognitive dimensions of prejudice. Personality and Social Psychology Bulletin, 31, 1145-1158.

Wilson, K. G. y Luciano, M. C. (2002). Terapia de aceptación y compromiso. Un tratamiento conductual orientado a los valores. Madrid: Pirámide. 